\title{
Prevalence and Possible Risk Factors of Low Bone Mineral Density in Untreated Female Patients with Systemic Lupus Erythematosus
}

\author{
Yi-Ning Sun, ${ }^{1}$ Xiu-Yuan Feng, ${ }^{1}$ Lan He, ${ }^{1}$ Ling-Xia Zeng, ${ }^{2}$ Zhi-Ming Hao, ${ }^{1}$ \\ Xiao-Hong Lv, ${ }^{1}$ and Dan Pu${ }^{1}$ \\ ${ }^{1}$ Department of Rheumatology, First Affiliated Hospital of the Medical School, Xian Jiaotong University, Xian, Shaanxi 710061, China \\ ${ }^{2}$ Department of Epidemiology and Biostatistics, School of Public Health, Xian Jiaotong University, Xian, Shaanxi 710061, China
}

Correspondence should be addressed to Lan He; xajdljn@126.com

Received 8 October 2014; Accepted 19 January 2015

Academic Editor: Hiroshi Tanaka

Copyright (c) 2015 Yi-Ning Sun et al. This is an open access article distributed under the Creative Commons Attribution License, which permits unrestricted use, distribution, and reproduction in any medium, provided the original work is properly cited.

\begin{abstract}
Systemic lupus erythematosus (SLE) is an autoimmune disease characterized by chronic inflammation. Different studies have shown decreased bone mineral density (BMD) in patients with SLE. The objective of this study was to investigate the prevalence and possible risk factors of low BMD in untreated female patients with SLE in Chinese population. A total of 119 untreated female patients with SLE were included. BMD was measured at lumbar spine and at total hip by dual-energy X-ray absorptiometry. The associations between decreased BMD and demographic variables, clinical variables, and bone metabolism variables were analyzed. These SLE patients had the following characteristics: mean age was $32.6 \pm 11.9$ years, mean disease duration was $22.1 \pm 34.5$ months, and mean SLEDAI was $11.4 \pm 5.4$. Osteopenia was present in $31.1 \%$ of the patients and osteoporosis in $8.5 \%$. A significant negative association between low density lipoprotein cholesterol (LDL-c) and BMD at the lumbar spine (correlation coefficient $=-0.242$; $P=0.023$ ) and total hip (correlation coefficient $=-0.259 ; P=0.019$ ) was shown. These results seem to indicate that increased LDL-c may be an important risk factor for low BMD at lumbar spine and total hip in untreated female SLE patients.
\end{abstract}

\section{Introduction}

Systemic lupus erythematosus (SLE) is an autoimmune disease characterized by chronic inflammation and multisystemic damage. Studies from different parts of the world have shown osteoporosis and low bone mineral density (BMD) in patients with SLE [1, 2], especially in female patients [3]. In several cross-sectional studies, osteopenia is found in $4 \%$ to $74 \%$ of SLE patients and osteoporosis in 3\% to $48 \%$ of patients with SLE [4]. The discrepancy in the prevalence between different studies may be due to different study design, sex, age, ethnicity, and disease activity, but, in general, a reduction in BMD was demonstrated in patients with SLE.

Osteoporosis is characterized by low bone mass and damaged structural integrity that lead to increased risk of bone fracture. Osteoporosis-related fragility fractures represent one of the most important complications that may occur in patients with SLE. A five-year prospective study determined the incidence of nonvertebral and vertebral fracture in Chinese SLE patients was 1.26 and 0.94 per 100 patient-years [5]. The prevalence of vertebral fracture in SLE ranges between $20 \%$ and 29\% [6]. These fractures may provoke disability and contribute to an important decrease in quality of life. Moreover, long-term studies have shown vertebral fractures were associated with increased morbidity and mortality [7].

Patients with SLE are at increased risk for bone loss and fractures for many reasons. It has been established that the old age, postmenopausal status, and low body mass index are the possible risk factors for osteoporosis in SLE. Furthermore, chronic inflammation, immobility, and vitamin D deficiency due to lack of sun exposure and treatment with glucocorticoids may be common factors that substantially increase osteoporosis risk in these patients $[4,8]$. The main objective of this study was therefore to investigate the incidence of osteoporosis and osteopenia in untreated female patients 
with SLE by using dual-energy X-ray absorptiometry (DXA) and to identify the possible risk factors associated with low BMD in the SLE patients.

\section{Materials and Methods}

2.1. Patients. One hundred and nineteen untreated female patients with SLE were included in this study from August 2012 to August 2014. All patients attended the rheumatology clinic of the First Affiliated Hospital of the Medical School, Xian Jiaotong University. All patients fulfilled the 1997 American College of Rheumatology (ACR) revised criteria for the classification of SLE [9] and were provided written consent for participation. This study was approved by the Ethics Committee of the hospital.

2.2. BMD Measurements. BMD at the lumbar spine (anteroposterior, L1-L4) and total hip (left femoral neck) were performed by a single trained technician using dual-energy X-ray absorptiometry (LEXXOS-LX381, French DMS). All measurements were performed in accordance with standard instrument procedures and matched gender and weight. The precisions for duplicate measurements were $0.16 \%$ for lumbar spine and $0.15 \%$ for total hip. All BMD results were expressed in absolute values $\left(\mathrm{g} / \mathrm{cm}^{2}\right)$ and as the number of standard deviations (SD) above or below the mean results of young female adults, $T$ score, and compared with an age matched female reference population, $Z$ score. In this study, osteoporosis was defined as a $T$ score or $Z$ score $\leq-2.5$ in lumbar spine or total hip and osteopenia as a $T$ score or $Z$ score $<-1$ but $>-2.5$.

2.3. Clinical Data Collection and Analysis. Demographic data collected at the time of study inclusion were age, body weight, height, body mass index (BMI), menstrual status, and age at menopause. In addition, we inquired about lifestyle related data including smoking, intake of alcohol, history of lumbar or femoral head fracture, and family history of osteoporosis and fracture.

Clinical data of SLE patients were assessed for disease duration, serum albumin, serum creatinine, 24-hour urinary protein, serum levels of total cholesterol (TC), triglyceride, low density lipoprotein cholesterol (LDL-c), erythrocyte sedimentation rate (ESR), and C-reactive protein (CRP). Immunologic measures were regarding complement components and anti-double stranded DNA antibodies (anti-dsDNA antibodies). Disease activity was scored using the Systemic Lupus Erythematosus Disease Activity Index (SLEDAI) by the physicians in charge of the patients [10].

Biochemical and hormonal variables related to bone mineral metabolism such as serum levels of calcium, phosphate, and parathyroid hormone (PTH), levels of 25hydroxyvitamin D $(25[\mathrm{OH}] \mathrm{D})$, bone formation marker (Osteocalcin, OC), and bone resorption marker (C-terminal cross-linking telopeptide of type I collagen, CTX) were examined. All venous blood samples were taken after a one-night fast and analyzed consecutively using standard laboratory techniques in the Department of Clinical Laboratory at our hospital. Based on the laboratory reference value, deficiency of $25(\mathrm{OH}) \mathrm{D}$ was defined as a serum level $<20 \mathrm{ng} /$ milliliter. In female, a high titer of CTX was defined as a serum level $>573 \mathrm{pg} /$ milliliter during premenopause and $>1008$ pg/milliliter during postmenopause.

2.4. Statistical Analysis. SPSS 16.0 for Windows statistical software was used for data analysis. Measurement data were presented as the mean \pm standard deviation (mean \pm SD). The Student $t$-test and Mann-Whitney $U$ test were used to analyze parametric and nonparametric variables. Variables possibly associated with a decreased BMD at the lumbar spine and at the hip were examined by Pearson's correlation coefficients or Spearman's correlation coefficients. The following variables were examined in relationship to BMD: age, BMI, disease duration, disease activity, serum albumin, serum creatinine, 24-hour urine protein, serum TC, triglyceride and LDL-c, ESR, CRP, complement components, anti-dsDNA antibodies, serum calcium, phosphate, PTH, serum $25[\mathrm{OH}] \mathrm{D}, \mathrm{OC}$, and CTX. A $P$ value less than or equal to 0.05 (2-sided) was considered statistically significant.

\section{Results}

3.1. Demographic, Clinical, and Bone Metabolism Variables in SLE Patients. One hundred and nineteen untreated female SLE patients were included in the study. The demographic, clinical, and bone metabolism characteristics of the patients are shown in Table 1 . The median age of the participants was $32.6 \pm 11.9$ (10 67) yrs. Fourteen patients (11.9\%) were postmenopausal and the median age at menopause was $48.2 \pm$ 1.9 (45 51) yrs. The mean body mass index (BMI) of the patients was $20.6 \pm 3.3 \mathrm{~kg} / \mathrm{m}^{2}$. Mean disease duration was $22.1 \pm 34.5$ months and mean SLEDAI was $11.4 \pm 5.4$.

3.2. BMD in SLE Patients. The results of the BMD measurements are shown in Table 2. The mean BMD \pm SD was $0.91 \pm 0.16 \mathrm{~g} / \mathrm{cm}^{2}$ at the lumbar spine and $0.88 \pm 0.12 \mathrm{~g} / \mathrm{cm}^{2}$ at total hip. The mean $T$ score \pm SD was $-0.45 \pm 1.40$ at the lumbar spine and $-0.01 \pm 1.08$ at total hip. The frequency of osteopenia ( $T$ score or $Z$ score less than $-1.0 \mathrm{SD}$ at the lumbar spine [L1-L4] and/or at the total hip) was $31.1 \%$. The frequency of osteoporosis ( $T$ score or $Z$ score less than $-2.5 \mathrm{SD}$ at the lumbar spine [L1-L4] and/or at the total hip) was $8.5 \%$.

3.3. Characteristics of Patients with Low BMD. We further analyzed and compared the SLE patients with normal or low BMD at the lumbar spine or at total hip, as shown in Table 3. Serum total cholesterol was significantly increased in patients with low BMD $(4.2 \pm 1.8 \mathrm{mmol} /$ liter $)$ than those in normal BMD $(3.3 \pm 0.9 \mathrm{mmol} / \mathrm{liter}, P=0.007)$. Serum low density lipoprotein cholesterol was also increased in patients with low BMD $(2.4 \pm 1.2 \mathrm{mmol} /$ liter $)$ than those in normal $\operatorname{BMD}(1.9 \pm 0.6 \mathrm{mmol} / \mathrm{liter})$. However, the difference was not statistically significant $(P=0.055)$. 
TABLE 1: Demographic, clinical data and bone metabolic variables in the SLE patients.

\begin{tabular}{|c|c|}
\hline Variable & $\begin{array}{l}\text { All SLE patients } \\
\quad(n=119)\end{array}$ \\
\hline \multicolumn{2}{|l|}{ Demographic variables } \\
\hline Age, mean \pm SD years & $32.6 \pm 11.9$ \\
\hline Body mass index, mean $\pm \mathrm{SD} \mathrm{kg} / \mathrm{m}^{2}$ & $20.6 \pm 3.3$ \\
\hline Postmenopausal, \% & 11.9 \\
\hline Menopausal age, mean \pm SD years & $48.2 \pm 1.9$ \\
\hline \multicolumn{2}{|l|}{ Clinical variables } \\
\hline Disease duration, mean \pm SD months & $22.1 \pm 34.5$ \\
\hline Serum creatinine, mean $\pm \mathrm{SD} \mu \mathrm{mol} /$ liter & $53.9 \pm 23.3$ \\
\hline Serum albumin, mean \pm SD g/liter & $31.7 \pm 6.9$ \\
\hline 24-hour urine protein, mean \pm SD g/24 hr & $0.58 \pm 0.84$ \\
\hline Serum total cholesterol, mean \pm SD mmol/liter & $3.7 \pm 1.4$ \\
\hline Serum triglyceride, mean \pm SD mmol/liter & $2.0 \pm 1.3$ \\
\hline Serum low density lipoprotein cholesterol, mean \pm SD mmol/liter & $2.1 \pm 0.9$ \\
\hline Erythrocyte sedimentation rate, mean $\pm \mathrm{SD} \mathrm{mm} /$ hour & $53.8 \pm 34.7$ \\
\hline C-reactive protein, mean \pm SD mg/liter & $11.1 \pm 13.4$ \\
\hline Complement 3, mean \pm SD g/liter & $0.57 \pm 0.31$ \\
\hline Complement 4 , mean \pm SD g/liter & $0.10 \pm 0.09$ \\
\hline Anti-dsDNA antibodies, mean $\pm \mathrm{SD}$, IU/milliliter & $43.7 \pm 37.9$ \\
\hline SLEDAI, mean \pm SD & $11.4 \pm 5.4$ \\
\hline \multicolumn{2}{|l|}{ Bone metabolism variables } \\
\hline 25-Hydroxyvitamin $\mathrm{D}$, mean \pm SD ng/milliliter & $11.9 \pm 7.3$ \\
\hline Serum calcium, mean \pm SD mmol/liter & $2.0 \pm 0.2$ \\
\hline Serum phosphate, mean \pm SD mmol/liter & $1.1 \pm 0.3$ \\
\hline Parathyroid hormone $(\mathrm{PTH})$, mean $\pm \mathrm{SD}$ pg/milliliter & $36.2 \pm 22.9$ \\
\hline Osteocalcin $(\mathrm{OC})$, mean \pm SD ng/milliliter & $10.3 \pm 6.7$ \\
\hline $\begin{array}{l}\text { C-terminal cross-linking telopeptide of type I collagen (CTX), } \\
\text { mean } \pm \text { SD pg/milliliter }\end{array}$ & $557.3 \pm 396.2$ \\
\hline
\end{tabular}

SLE = systemic lupus erythematosus; SLEDAI = Systemic Lupus Erythematosus Disease Activity Index (range 0-105).

3.4. Variables Associated with Low Bone Mineral Density. To reveal the risk factors that may relate to the low BMD, we analyzed the demographic, clinical, and bone metabolism variables in patients with abnormal BMD as shown in Table 4. We found that serum low density lipoprotein cholesterol correlated negatively with low BMD at the lumbar spine (correlation coefficient $=-0.242, P=0.023$ ) and at the hip significantly (correlation coefficient $=-0.259, P=0.019$ ). However, we found no significant correlation between serum total cholesterol and low BMD at lumbar spine and at total hip. BMD at the lumbar spine and the hip was not associated with age, BMI, disease duration, disease activity, ESR, CRP, and complement components. Serum 25[OH]D was considered and analyzed as a potential risk factor for reduced $\mathrm{BMD}$, but no statistically significant correlation was found.

\section{Discussion}

In patients with systemic lupus erythematosus (SLE), an increase in bone loss and fragility fracture was found compared with general population [11]. Recent studies have shown a prevalence of osteopenia of $28-46 \%$ and a prevalence of osteoporosis of 3-9\% among SLE patients [12, 13]. The discrepancy among the different studies may be due to sex, ages, menopausal status, ethnicity, different disease duration, and different disease activity. For example, BMD in postmenopausal patients with SLE (osteopenia 74\%; osteoporosis $48 \%$ ) was more dramatically decreased than that in premenopausal patients (osteopenia 40\%; osteoporosis 5\%) $[2,14]$. Racial difference in the prevalence of low BMD in SLE patients may exist. Lee et al. [15] found that African American women had lower BMD at the hip and lumbar spine compared with white women with SLE. Three studies reported the prevalence of osteopenia in $30 \%$ to $50 \%$ and osteoporosis in $13.8 \%$ to $48 \%$ of Asian female SLE patients [1, $14,16]$. We demonstrated a prevalence of osteopenia of $31.1 \%$ and a prevalence of osteoporosis of $8.5 \%$ among untreated female SLE patients. Our findings were in consistency with previous studies of SLE patients, showing reduced bone mass at lumbar spine and at the hip in SLE patients.

Interestingly, the higher proportion of osteopenia and osteoporosis at the lumbar spine $(25.5 \% ; 10.4 \%)$ when compared with the hip $(7.5 \%$; $0.9 \%)$ was observed in our study 
TABLE 2: Lumbar spine and total hip BMD in the study of SLE patients.

\begin{tabular}{lc}
\hline Variable & $\begin{array}{c}\text { All SLE patients } \\
(n=119)\end{array}$ \\
\hline BMD, mean \pm SD g/cm ${ }^{2}$ & $0.91 \pm 0.16$ \\
Spine L1-L4 & $0.88 \pm 0.12$ \\
Total hip & \\
$T$ score, mean \pm SD & $-0.45 \pm 1.40$ \\
$\quad$ Spine L1-L4 & $-0.01 \pm 1.08$ \\
Total hip & \\
$Z$ score, mean \pm SD & $-0.36 \pm 1.34$ \\
Spine L1-L4 & $0.62 \pm 1.03$ \\
Total hip & \\
Osteopenia, \% & 31.1 \\
Lumbar spine or/and total hip & 25.5 \\
Lumbar spine & 10.4 \\
Total hip & \\
Osteoporosis, \% & 8.5 \\
Lumbar spine or/and total hip & 7.5 \\
Lumbar spine & 0.9 \\
Total hip &
\end{tabular}

SLE = systemic lupus erythematosus; BMD = bone mineral density.

(Table 2). The reasons for this discrepancy are unclear but may to some extent be related to variations in the type of bone comprising these two anatomic sites [17]. Glucocorticoids may be considered an important factor to induce bone loss in SLE patients, as glucocorticoids can directly inhibit osteoblasts and thus reduce the bone formation [18]. The lumbar spine, which is composed predominantly of trabecularrich bone, is more susceptible to the adverse effects of corticosteroids than the hip that consists of comparatively less trabecular bone. In the present study, untreated SLE patients were included in order to exclude glucocorticoids exposure which may influence BMD measurements. In fact, studies have observed trabecular bone loss in patients with SLE that could not be explained by corticosteroids use [19]. Apart from glucocorticoids, a rapid loss of spine trabecular bone in untreated SLE patients is likely to arise from chronic inflammation and disease damage [20] and several studies have shown the high prevalence of vertebral fractures in SLE patients [6, 21-23]. Cross-sectional studies have found that low BMD at the hip remained as a significant factor for the presence of vertebral fracture $[6,22]$. In fact, osteoporosis at the lumbar spine may be one of the contribution factors associated with both new vertebral and nonvertebral fractures [5].

Various risk factors and clinical variables have been demonstrated to associate with decreased BMD in the spine and hip of SLE patients $[4,8]$. In our study of untreated female SLE patients, we found a significant negative correlation between low BMD at the lumbar spine (correlation coefficient $=-0.242, P=0.023$ ) and at total hip (correlation coefficient $=-0.259, P=0.019)$ and serum LDL-c, indicating the
TABLE 3: Characteristics of SLE patients with normal and low BMD at lumbar spine and total hip.

\begin{tabular}{|c|c|c|c|}
\hline Variable & Normal BMD & Low BMD & $P$ \\
\hline \multicolumn{4}{|l|}{ Demographic variables } \\
\hline Age $^{\#}$ & $31.6 \pm 8.9$ & $33.9 \pm 16.1$ & 0.869 \\
\hline $\mathrm{BMI}^{\#}$ & $20.7 \pm 3.5$ & $20.3 \pm 2.8$ & 0.730 \\
\hline \multicolumn{4}{|l|}{ Clinical variables } \\
\hline Disease duration $^{\#}$ & $24.1 \pm 34.7$ & $20.1 \pm 34.5$ & 0.895 \\
\hline Serum creatinine ${ }^{\#}$ & $54.6 \pm 26.7$ & $55.2 \pm 20.3$ & 0.651 \\
\hline Serum albumin ${ }^{\#}$ & $31.7 \pm 5.8$ & $32.3 \pm 8.6$ & 0.216 \\
\hline 24 -hour urine protein ${ }^{\#}$ & $0.56 \pm 0.75$ & $0.62 \pm 1.02$ & 0.586 \\
\hline Serum TC ${ }^{\#}$ & $3.3 \pm 0.9$ & $4.2 \pm 1.8$ & 0.007 \\
\hline Serum triglyceride ${ }^{\#}$ & $2.0 \pm 1.1$ & $2.0 \pm 1.7$ & 0.332 \\
\hline Serum LDL-c ${ }^{\#}$ & $1.9 \pm 0.6$ & $2.4 \pm 1.2$ & 0.055 \\
\hline $\mathrm{ESR}^{\#}$ & $56.1 \pm 34.7$ & $54.9 \pm 33.0$ & 0.948 \\
\hline $\mathrm{CRP}^{\#}$ & $10.8 \pm 12.9$ & $10.8 \pm 12.7$ & 0.963 \\
\hline Complement $3^{\#}$ & $0.57 \pm 0.32$ & $0.59 \pm 0.33$ & 0.892 \\
\hline Complement $4^{\#}$ & $0.10 \pm 0.09$ & $0.10 \pm 0.09$ & 0.909 \\
\hline SLEDAI $^{\#}$ & $11.7 \pm 6.0$ & $10.8 \pm 5.2$ & 0.579 \\
\hline \multicolumn{4}{|l|}{ Bone metabolism variables } \\
\hline Serum 25-[OH] $\mathrm{D}^{\#}$ & $12.2 \pm 7.8$ & $11.8 \pm 6.7$ & 0.995 \\
\hline Serum calcium ${ }^{*}$ & $2.0 \pm 0.2$ & $2.0 \pm 0.2$ & 0.978 \\
\hline Serum phosphate* & $1.2 \pm 0.3$ & $1.0 \pm 0.3$ & 0.715 \\
\hline $\mathrm{PTH}^{\#}$ & $34.8 \pm 23.7$ & $38.1 \pm 21.3$ & 0.342 \\
\hline $\mathrm{OC}^{\#}$ & $10.1 \pm 5.5$ & $11.2 \pm 7.9$ & 0.880 \\
\hline $\mathrm{CTX}^{\#}$ & $500.8 \pm 357.8$ & $633.7 \pm 498.0$ & 0.373 \\
\hline
\end{tabular}

*The Student $t$-test; ${ }^{*}$ Mann-Whitney $U$ test.

$\mathrm{BMD}=$ bone mineral density; $\mathrm{BMI}=$ body mass index; $\mathrm{TC}=$ total cholesterol; LDL-c = low density lipoprotein cholesterol; ESR = erythrocyte sedimentation rate; $\mathrm{CRP}=\mathrm{C}$-reactive protein; SLEDAI $=$ Systemic Lupus Erythematosus Disease Activity Index; 25- $[\mathrm{OH}] \mathrm{D}=25$-hydroxyvitamin D; PTH $=$ parathyroid hormone; $\mathrm{OC}=$ osteocalcin; $\mathrm{CTX}=\mathrm{C}$-terminal crosslinking telopeptide of type I collagen.

importance of increased LDL-c as a major risk factor for low BMD at lumbar spine and at total hip. We could not find the correlation between BMI, disease activity, 25(OH)D, ESR, CRP, complement components, and low BMD at the lumbar spine and at the hip. These findings were consistent with two large-population studies in Indian and Korean population, which identified that LDL-c and TC had weak but significant negative correlation with BMD at lumbar spine and at the hip in men and pre- and postmenopausal women [23, 24]. A lower fat intake and body fatness as well as a better profile of LDL-c predicted high BMD in healthy women [25].

Although atherosclerosis and osteoporosis are considered to be different in pathology, they share bidirectional correlation [26]. On the one hand, low bone density is associated with incident cardiovascular disease [27]. On the other hand, women with cardiovascular disease have increased risk of osteoporotic fracture [28]. In patients with SLE, decreased $\mathrm{BMD}$ is an independent predictor for premature coronary calcification [29]. The definite link between atherosclerosis and osteoporosis suggested that they may share common risk 
TABLE 4: Relationship between BMD at lumbar spine and total hip and demographic, clinical, and bone metabolism variables by univariate analysis.

\begin{tabular}{|c|c|c|c|c|}
\hline \multirow{2}{*}{ Variable } & \multicolumn{2}{|c|}{ BMD in lumbar spine } & \multicolumn{2}{|c|}{ BMD in total hip } \\
\hline & Correlation coefficient & $P$ & Correlation coefficient & $P$ \\
\hline \multicolumn{5}{|l|}{ Demographic variables } \\
\hline $\operatorname{Age}^{\#}$ & 0.028 & 0.776 & -0.153 & 0.136 \\
\hline BMI* $^{*}$ & 0.051 & 0.603 & 0.160 & 0.119 \\
\hline \multicolumn{5}{|l|}{ Clinical variables } \\
\hline Disease duration ${ }^{\#}$ & -0.036 & 0.717 & -0.077 & 0.457 \\
\hline Serum albumin & -0.166 & 0.091 & -0.088 & 0.395 \\
\hline Serum creatinine $e^{\#}$ & -0.041 & 0.677 & -0.167 & 0.104 \\
\hline 24-hour urine protein ${ }^{\#}$ & -0.020 & 0.856 & -0.068 & 0.561 \\
\hline Serum $\mathrm{TC}^{\#}$ & -0.193 & 0.052 & -0.135 & 0.198 \\
\hline Serum triglyceride ${ }^{\#}$ & 0.129 & 0.228 & -0.009 & 0.936 \\
\hline Serum LDL-c ${ }^{*}$ & -0.242 & 0.023 & -0.259 & 0.019 \\
\hline $\mathrm{ESR}^{\#}$ & -0.084 & 0.401 & -0.013 & 0.905 \\
\hline $\mathrm{CRP}^{\#}$ & -0.089 & 0.401 & -0.085 & 0.439 \\
\hline Complement $3^{\#}$ & -0.084 & 0.395 & 0.033 & 0.751 \\
\hline Complement $4^{\#}$ & -0.068 & 0.493 & -0.095 & 0.361 \\
\hline SLEDAI $^{\#}$ & 0.012 & 0.907 & 0.109 & 0.290 \\
\hline \multicolumn{5}{|l|}{ Bone metabolism variables } \\
\hline Serum $25-[\mathrm{OH}] \mathrm{D}^{\#}$ & -0.024 & 0.810 & 0.094 & 0.371 \\
\hline Serum calcium* & -0.125 & 0.215 & 0.094 & 0.373 \\
\hline Serum phosphate* & 0.130 & 0.216 & 0.187 & 0.089 \\
\hline $\mathrm{PTH}^{\#}$ & -0.147 & 0.154 & -0.162 & 0.128 \\
\hline $\mathrm{OC}^{\#}$ & -0.055 & 0.588 & 0.011 & 0.916 \\
\hline $\mathrm{CTX}^{\#}$ & -0.152 & 0.170 & -0.163 & 0.164 \\
\hline
\end{tabular}

*Pearson's correlation coefficients; ${ }^{\#}$ Spearman's correlation coefficients.

$\mathrm{BMD}=$ bone mineral density; $\mathrm{BMI}=$ body mass index; $\mathrm{TC}=$ total cholesterol; LDL-c $=$ low density lipoprotein cholesterol; ESR = erythrocyte sedimentation rate; $\mathrm{CRP}=\mathrm{C}$-reactive protein; SLEDAI = Systemic Lupus Erythematosus Disease Activity Index; 25 - $[\mathrm{OH}] \mathrm{D}=25$-hydroxyvitamin $\mathrm{D}$; PTH = parathyroid hormone; $\mathrm{OC}=$ osteocalcin; $\mathrm{CTX}=\mathrm{C}$-terminal cross-linking telopeptide of type I collagen.

factors. Oxidized LDL is well known to play an important role in the generation and progression of atherosclerosis. So it may be considered as a candidate that links both disorders. In fact, it has been shown that high serum LDLc level may be a risk factor for low BMD [23] and for nonvertebral fragility fracture [30]. Increased level of oxidized LDL-c was demonstrated in patients with active lupus and chronic systemic inflammation including dyslipidemia may contribute to the bone loss in SLE [31]. Experiments in mice have shown that oxidized lipid may attenuate osteogenesis and parathyroid hormone bone anabolism is blunted in hyperlipidemic mice [32]. Oxidized LDL may negatively influence bone formation by reducing osteoblast maturation and phosphate-induced mineralization in osteoblasts [33]. Oxidized LDL increases the expression of receptor activator of nuclear factor kappa-B ligand (RANKL) on osteoblast via extracellular signal regulated kinase (ERK), nuclear factor $\kappa \mathrm{B}(\mathrm{NF} \kappa \mathrm{B})$, and nuclear factor of activated-T-cell (NFAT) signaling pathways [34]. RANKL can stimulate osteoclast survival, activation, and differentiation, so it is conceivable to infer that increased level of RANKL may cause a decrease in BMD. In addition, since RANKL is involved in myocardial inflammation, vascular calcification, and plaque rupture, the increase in RANKL in prooxidant conditions observed in endothelial cells and fibroblasts may link the atherosclerosis to osteoporosis [35].

In conclusion, we measured the BMD at lumbar spine and at total hip of 119 untreated female patients with SLE. The incidences of osteopenia and osteoporosis in these patients were $31.1 \%$ and $8.5 \%$, respectively. We found that the patients with low BMD had increased LDL-c level in the serum and that increased LDL-c correlates negatively with low BMD at the lumbar spine and at total hip. Our results reveal a new risk factor for the bone loss in SLE and may shed some light on the future prevention and treatment of osteopenia and osteoporosis in female SLE patients.

\section{Conflict of Interests}

The authors declare that there is no conflict of interests regarding the publication of this paper.

\section{Acknowledgments}

The authors are grateful to Dr. Ling-Xia Zeng for statistical advice and support. They thank nurses and graduated 
students in their department for their assistance with the patients. This study was supported by grants from Xian Jiaotong University for clinical research. Xiu-Yuan Feng is a co-first author.

\section{References}

[1] M. Furukawa, C. Kiyohara, H. Tsukamoto et al., "Prevalence of and risk factors for low bone mineral density in Japanese female patients with systemic lupus erythematosus," Rheumatology International, vol. 31, no. 3, pp. 365-376, 2011.

[2] C. Mendoza-Pinto, M. García-Carrasco, H. Sandoval-Cruz et al., "Risks factors for low bone mineral density in premenopausal Mexican women with systemic lupus erythematosus," Clinical Rheumatology, vol. 28, no. 1, pp. 65-70, 2009.

[3] K. Almehed, E. H. Forsblad d', G. Kvist, C. Ohlsson, and H. Carlsten, "Prevalence and risk factors of osteoporosis in female SLE patients-extended report," Rheumatology, vol. 46, no. 7, pp. 1185-1190, 2007.

[4] M. García-Carrasco, C. Mendoza-Pinto, R. O. Escárcega et al., "Osteoporosis in patients with systemic lupus erythematosus," Israel Medical Association Journal, vol. 11, no. 8, pp. 486-491, 2009.

[5] T. Y. Zhu, J. F. Griffith, S.-K. Au et al., "Incidence of and risk factors for non-vertebral and vertebral fracture in female Chinese patients with systemic lupus erythematosus: a five-year cohort study," Lupus, vol. 23, no. 9, pp. 854-861, 2014.

[6] K. Almehed, S. Hetényi, C. Ohlsson et al., "Prevalence and risk factors of vertebral compression fractures in female SLE patients," Arthritis Research and Therapy, vol. 12, no. 4, pp. R153R159, 2010.

[7] M. García-Carrasco, C. Mendoza-Pint, C. Riebeling et al., "Influence of prevalent vertebral fractures on the quality of life of patients with systemic lupus erythematosus," Israel Medical Association Journal, vol. 13, no. 6, pp. 333-337, 2011.

[8] I. E. M. Bultink, M. Vis, I. E. van der Horst-Bruinsma, and W. F. Lems, "Inflammatory rheumatic disorders and bone," Current Rheumatology Reports, vol. 14, no. 3, pp. 224-230, 2012.

[9] M. C. Hochberg, "Updating the American College of Rheumatology revised criteria for the classification of systemic lupus erythematosus," Arthritis and rheumatism, vol. 40, no. 9, article 1725, 1997.

[10] C. Bombardier, D. D. Gladman, M. B. Urowitz et al., "Derivation of the SLEDAI: a disease activity index for lupus patients," Arthritis and Rheumatism, vol. 35, no. 6, pp. 630-640, 1992.

[11] C.-S. Yee, N. Crabtree, J. Skan et al., "Prevalence and predictors of fragility fractures in systemic lupus erythematosus," Annals of the Rheumatic Diseases, vol. 64, no. 1, pp. 111-113, 2005.

[12] T. C. Salman-Monte, V. Torrente-Segarra, J. Muñoz-Ortego, S. Mojal, and J. Carbonell-Abelló, "Prevalence and predictors of low bone density and fragility fractures in women with systemic lupus erythematosus in a Mediterranean region," Rheumatology International, 2014.

[13] J. Jacobs, L.-A. Korswagen, A. M. Schilder et al., "Six-year follow-up study of bone mineral density in patients with systemic lupus erythematosus," Osteoporosis International, vol. 24, no. 6, pp. 1827-1833, 2013.

[14] C. C. Mok, A. Mak, and K. M. Ma, "Bone mineral density in postmenopausal Chinese patients with systemic lupus erythematosus," Lupus, vol. 14, no. 2, pp. 106-112, 2005.
[15] C. Lee, O. Almagor, D. D. Dunlop et al., "Association between African American race/ethnicity and low bone mineral density in women with systemic lupus erythematosus," Arthritis Care and Research, vol. 57, no. 4, pp. 585-592, 2007.

[16] S. Banno, Y. Matsumoto, T. Naniwa et al., "Reduced bone mineral density in Japanese premenopausal women with systemic lupus erythematosus treated with glucocorticoids," Modern Rheumatology, vol. 12, no. 4, pp. 323-328, 2002.

[17] H. Chen and K. Y. Kubo, "Bone three-dimensional microstructural features of the common osteoporotic fracture sites," World Journal of Orthopedics, vol. 5, no. 4, pp. 486-495, 2014.

[18] R. M. R. Pereira, J. F. de Carvalho, and E. Canalis, "Glucocorticoid-induced osteoporosis in rheumatic diseases," Clinics, vol. 65, no. 11, pp. 1197-1205, 2010.

[19] C. A. Pineau, M. B. Urowitz, P. J. Fortin, D. Ibanez, and D. D. Gladman, "Osteoporosis in systemic lupus erythematosus: factors associated with referral for bone mineral density studies, prevalence of osteoporosis and factors associated with reduced bone density," Lupus, vol. 13, no. 6, pp. 436-441, 2004.

[20] Y. Tang, H. Xie, J. Chen et al., "Activated $N F-\kappa B$ in bone marrow mesenchymal stem cells from systemic lupus erythematosus patients inhibits osteogenic differentiation through downregulating smad signaling," Stem Cells and Development, vol. 22, no. 4, pp. 668-678, 2013.

[21] I. E. M. Bultink, W. F. Lems, P. J. Kostense, B. A. C. Dijkmans, and A. E. Voskuyl, "Prevalence of and risk factors for low bone mineral density and vertebral fractures in patients with systemic lupus erythematosus," Arthritis \& Rheumatism, vol. 52, no. 7, pp. 2044-2050, 2005.

[22] C. Mendoza-Pinto, M. García-Carrasco, H. Sandoval-Cruz et al., "Risk factors of vertebral fractures in women with systemic lupus erythematosus," Clinical Rheumatology, vol. 28, no. 5, pp. 579-585, 2009.

[23] M. K. Garg, R. K. Marwaha, N. Tandon, K. Bhadra, and N. Mahalle, "Relationship of lipid parameters with bone mineral density in Indian population," Indian Journal of Endocrinology and Metabolism, vol. 18, no. 3, pp. 325-332, 2014.

[24] I.-K. Jeong, S. W. Cho, S. W. Kim et al., "Lipid profiles and bone mineral density in pre- and postmenopausal women in korea," Calcified Tissue International, vol. 87, no. 6, pp. 507-512, 2010.

[25] K. S. Sarkis, L. A. Martini, V. L. Szejnfeld, and M. M. Pinheiro, "Low fatness, reduced fat intake and adequate plasmatic concentrations of LDL-cholesterol are associated with high bone mineral density in women: a cross-sectional study with control group," Lipids in health and disease, vol. 11, pp. 37-44, 2012.

[26] A. Fisher, W. Srikusalanukul, M. Davis, and P. Smith, "Cardiovascular diseases in older patients with osteoporotic hip fracture: prevalence, disturbances in mineral and bone metabolism, and bidirectional links," Clinical Interventions in Aging, vol. 8, pp. 239-256, 2013.

[27] G. N. Farhat, A. B. Newman, K. Sutton-Tyrrell et al., "The association of bone mineral density measures with incident cardiovascular disease in older adults," Osteoporosis International, vol. 18, no. 7, pp. 999-1008, 2007.

[28] J. S. Chen, C. Hogan, G. Lyubomirsky, and P. N. Sambrook, "Women with cardiovascular disease have increased risk of osteoporotic fracture," Calcified Tissue International, vol. 88, no. 1, pp. 9-15, 2011.

[29] G. G. Ribeiro, E. Bonfá, R. S. Neto et al., "Premature coronary artery calcification is associated with disease duration and bone mineral density in young female systemic lupus erythematosus patients," Lupus, vol. 19, no. 1, pp. 27-33, 2010. 
[30] M. Yamauchi, T. Yamaguchi, K. Nawata, K.-I. Tanaka, S. Takaoka, and T. Sugimoto, "Increased low-density lipoprotein cholesterol level is associated with non-vertebral fractures in postmenopausal women," Endocrine, 2014.

[31] J. Frostegård, E. Svenungsson, R. Wu et al., "Lipid peroxidation is enhanced in patients with systemic lupus erythematosus and is associated with arterial and renal disease manifestations," Arthritis and Rheumatism, vol. 52, no. 1, pp. 192-200, 2005.

[32] A. P. Sage, J. Lu, E. Atti et al., "Hyperlipidemia induces resistance to PTH bone anabolism in mice via oxidized lipids," Journal of Bone and Mineral Research, vol. 26, no. 6, pp. 1197-1206, 2011.

[33] C. Mazière, V. Savitsky, A. Galmiche, C. Gomila, Z. Massy, and J.-C. Mazière, "Oxidized low density lipoprotein inhibits phosphate signaling and phosphate-induced mineralization in osteoblasts. Involvement of oxidative stress," Biochimica et Biophysica Acta, vol. 1802, no. 11, pp. 1013-1019, 2010.

[34] C. Mazière, V. Salle, C. Gomila, and J.-C. Mazière, "Oxidized low density lipoprotein enhanced RANKL expression in human osteoblast-like cells. Involvement of ERK, NFkappaB and NFAT,' Biochimica et Biophysica Acta, vol. 1832, no. 10, pp. 1756-1764, 2013.

[35] C. Mazière, V. Salle, C. Gomila, and J.-C. Mazière, "Oxidized low density lipoprotein increases RANKL level in human vascular cells. Involvement of oxidative stress," Biochemical and Biophysical Research Communications, vol. 440, no. 2, pp. 295299, 2013. 


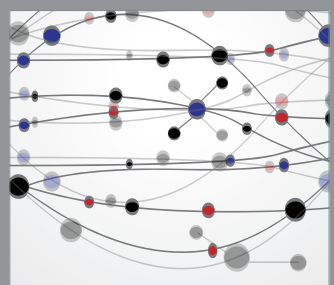

The Scientific World Journal
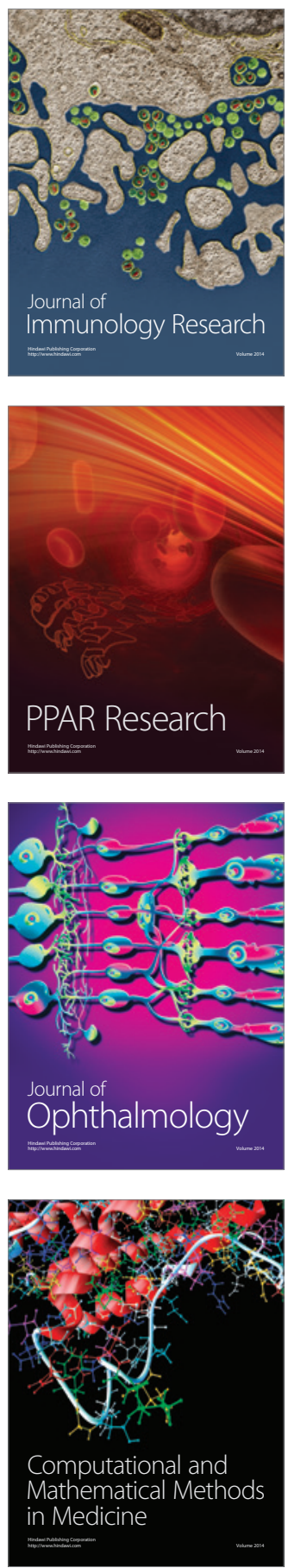

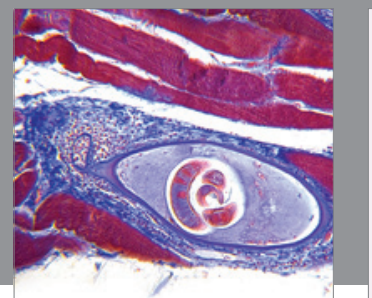

Gastroenterology

Research and Practice
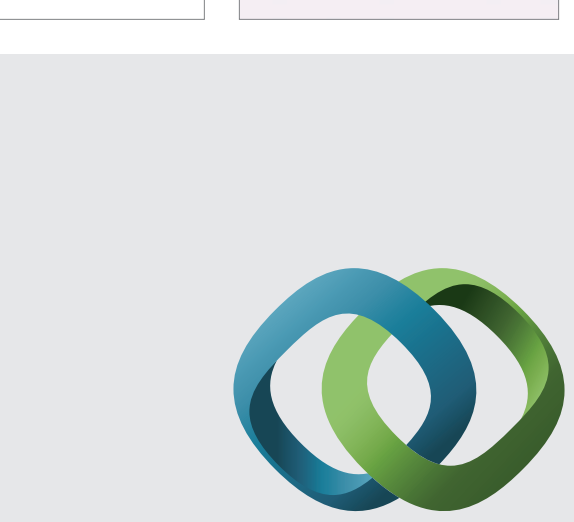

\section{Hindawi}

Submit your manuscripts at

http://www.hindawi.com
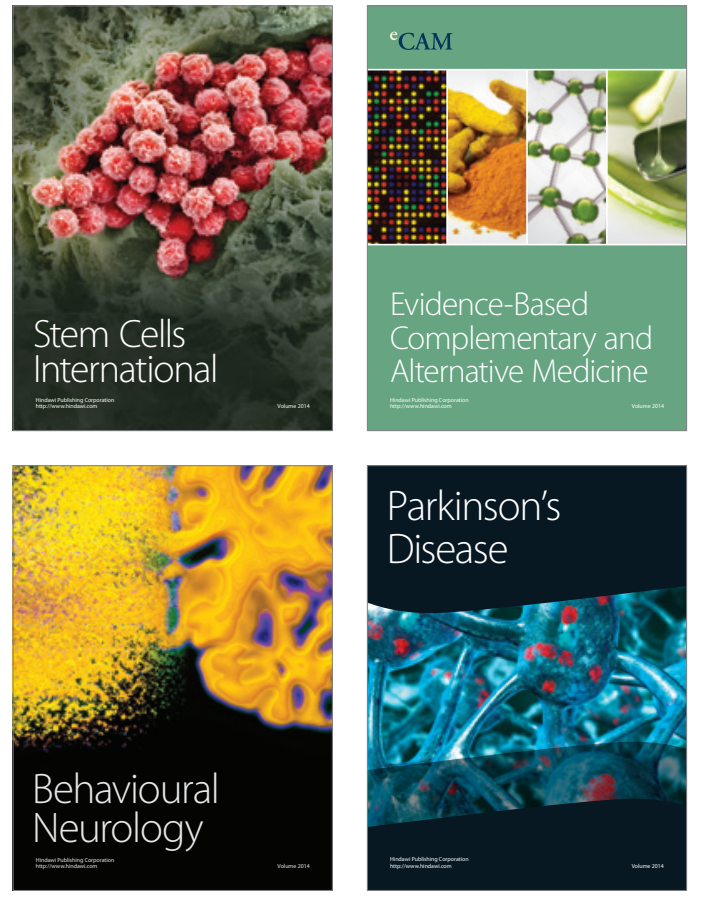
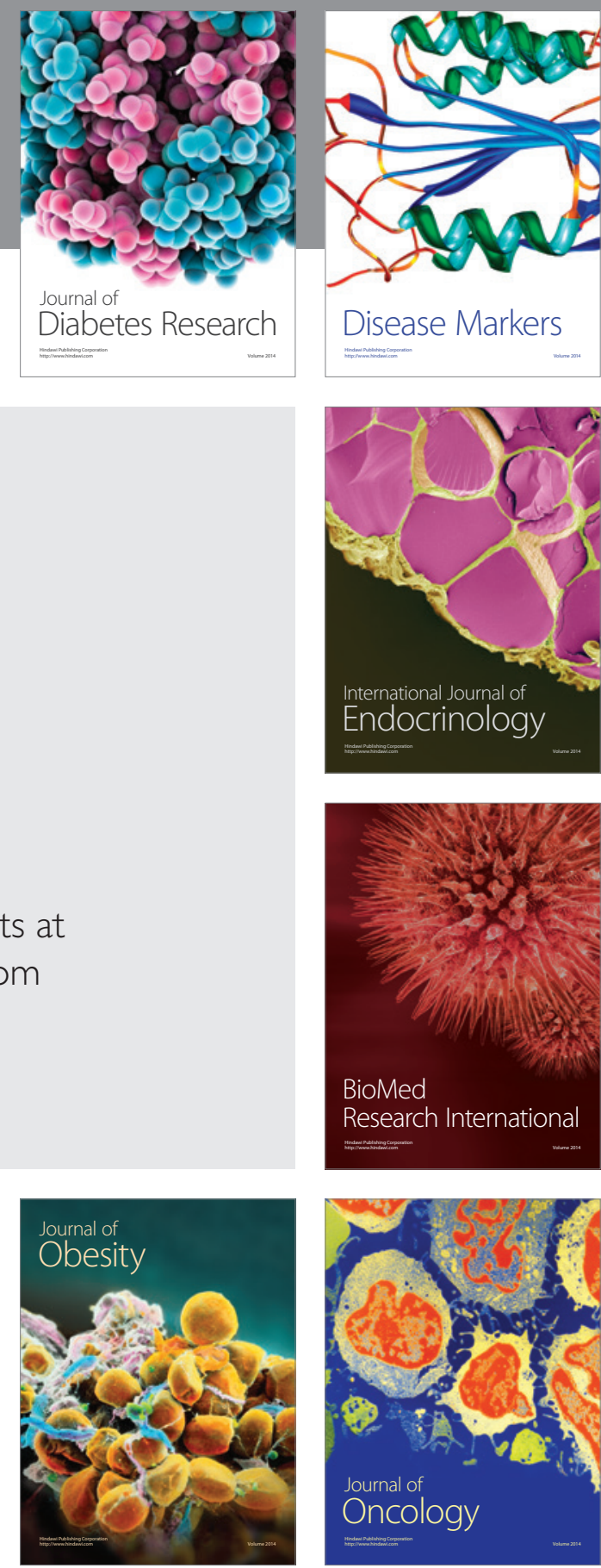

Disease Markers
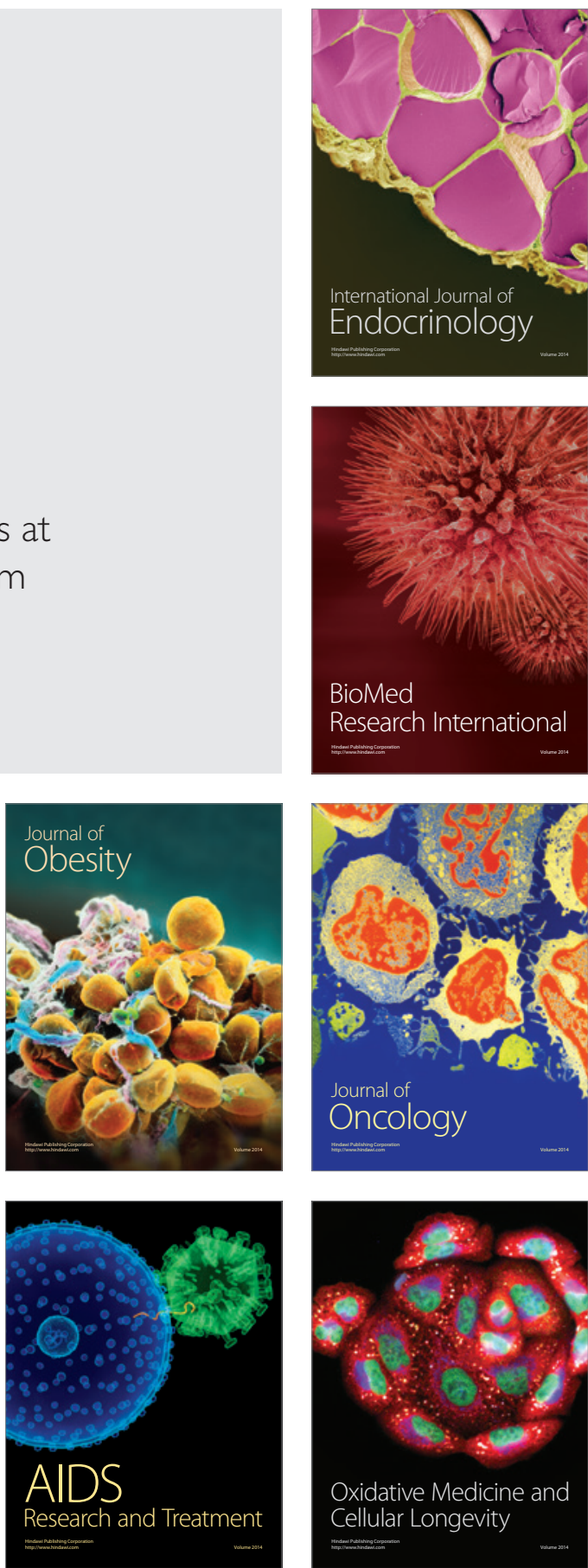